\title{
Einige Bemerkungen zu Amos.
}

Von Privatdocent Lic. Edwin Albert in Konigsberg i. Pr.

\section{Am 26.}

Gewöhnlich erklärt man, daß Amos hier die schlimme Rechtspflege tadele: die Richter lassen sich bestechen und verkaufen den uֵ Geld, das heißt, derjenige der im Recht ist, wird verurteilt, wenn der andere dem Richter das notige Geld in die Hand drückt. Die parallele

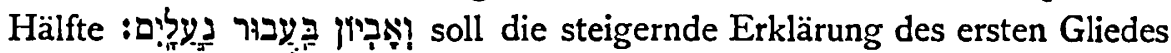
sein: zwei ganze Sandalen genügen schon zur Bestechung des Richters, um die Verurteilung des Unschuldigen von ihm zu erlangen ${ }^{x}$. Doch ist der Gedanke, man brauchte dem Richter statt Geld nur ein Paar Sandalen zu geben, damit er den Unschuldigen verurteilte, nicht gut möglich. Für Geld ist der Mann zu haben, auch für eine geringe Summe, aber doch nicht für ein Paar Sandalèn; das würde dem Sinn des Ganzen widersprechen; denn der Richter macht ja doch ein Geschäft aus seiner Stellung, das aber, wenn er sich schon mit ein Paar Sandalen oder deren Geldwert begnügt, nicht zu verstehen ist. Eher wäre dann der Ausdruck schon in der von HOFFMANN unten erwahnten Fassung möglich.

DUHM behauptet, hier sei nicht vom Richten die Rede, sondern vom Verkaufen, nicht von einem Verbrechen gegen das formale Recht, sondern von Vergehen gegen Billigkeit und Menschlichkeit ${ }^{2}$. Diese Deutung erschlieft DuHM allerdings erst auf Grund der Textänderung iș da jenes keine gute Parallele zu ș sei. Diese Erklärung DuHMs ist wohl als die allein richtige zu bezeichnen; da sie sich aber auf eine Konjektur gründet, die noch dazu durch keine Handschrift gestützt werden kann, so hat sie zunächst wenig Wahrscheinlichkeit fur sich.

I So MAKTI: Das Dodekapropheton I904 S. 166. Ähnlich NowaCk: Die kleinen Propheten 1903 S. 133. Im wesentlichen dieselbe Deutung gibt auch HoFrmanN (ZAW

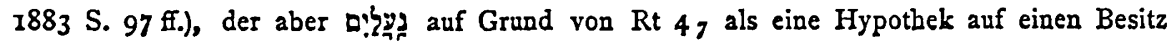
ansieht, mit der der Richter auch schon zufrieden ist und sich dadurch bestechen läbt, sein Amt za mißbrauchen.

2 ZAW I9IIS. $2 \mathrm{f}$. 
V. 7a, der eng zu v. 6 gehört, ist zwar verderbt, der Sinn aber trotzdem zu erkennen: Amos tadelt die Unmenschlichkeit derer, die die Gewalt in Händen haben, gegen die Armen, und zwar ist hier nicht von den Richtern, sondern von allen Israeliten die Rede, die so handeln ${ }^{x}$. So wenig nun in v. $7 \mathrm{a}$ Richter gemeint sind, so wenig ist in $6 \mathrm{~b}$ an sie gedacht. Nicht die Rechtsplege wird getadelt, sondern dab man den Pִ verkauft; dabei kann nur der Verkauf in die Sklaverei gemeint sein. Das Suff. in מִכְִ bezieht sich auf dieselben, die auch in v. 7 a getadelt werden, nämlich die Machthaber, denen der Arme überhaupt nichts gilt.

Der zweite Stichos ist entsprechend dem ersten zu fassen: „und Arme (verkaufen sie) um ein Paar Sandalen." ș ș stehen parallel und beide im Gegensatz zu denjenigen, welche die Macht, nämlich das Geld haben, und darum die Armen in jeder Weise unterdrücken.

Grundlos durfte man den Armen nicht verkaufen, nur wenn dieser in Schulden geraten war, die er nicht bezahlen konnte. Diesen Fall hat Amos im Auge. Die Gläubiger verkauften, um das ausgeliehene Geld zurückzuerhalten, die zahlungsunfabigen Schuldner in die Sklaverei und nahmen dabei keine Rücksicht auf die Person; - es war ihnen ganz gleich, ob sie es mit einem $p$ pa tun hatten, einem rechtschaffenen, frommen Mann, der vielleicht ohne eigene Schuld in Not geraten war, die Hauptsache war ihnen ihr Geld und ob der Mann zahlen konnte oder nicht. Darum war es diesen geldgierigen Leuten auch gleich, um wieviel es sich handelte; auch eine geringe Schuld, und war es nur der Wert, den etwa ein Paar Sandalen hatten, wulten sie auf diese Weise zurückzuerhalten. Amos tadelt hiermit also die Wucherer, die Kapitalisten, denen das Geld alles war und der Schuldner nur Ware, selbst wenn es sich dabei um einen, Sִִִ handelte. So verurteilt der Prophet hier das Treiben der Glaubiger, die sich gar kein Gewissen daraus machen, so lieblos, ja unmenschlich mit ihren Schuldnern zu verfahren. Somit stellt Amos hier die Pflicht der Nächstenliebe gegenüber den Glaubensgenossen uber die Bestimmungen des Schuldrechts.

Der dem pas parallele Ausdruck bereitet bei dieser Fassung keine Schwierigkeiten, da das Wort erstens in der allgemeinen Bedeutung "fromm, rechtschaffen" häufig ist (vgl. GesENIUS-BUHL) und zweitens schon bei Amos mit seinem Eifern gegen die Reichen und allen Luxus sich die Wurzeln der Anschauung finden, daß der von den Reichen unterdrückte Arme ein Pִִָ ist, jene dagegen die Gottlosen, Ungerechten, Sünder usw. darstellen.

I So auch MARTI a. a. O. S. 167. 
$29 \mathrm{~b}$.

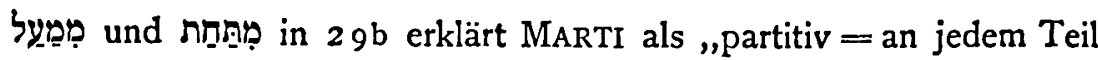
des Oben oder Unten" (S. I69). Diese Zusammensetzung von Pràpositionen hat jedoch hier nicht diese partitive Bedeutung, sondern entspricht dem regelmäßigen hebräischen Sprachgebrauch. Die Früchte und Wurzeln befinden sich לפַn bezw. unten weg. So werden z. B. die Feinde, die sich לִכִ befinden, ver-

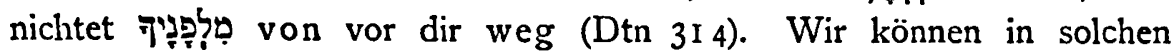
Fällen die Präposition nicht gut wiedergeben. Vgl. dazu das Material bei Gesenius-Buhl.

$$
2 \text { 10-12 (5 25). }
$$

MARTI gibt 2 Io wie auch $525 \mathrm{zu}$, dab Amos die Tradition von dem vierzigjährigen Aufenthalt in der Wüste gekannt haben mag, und streicht doch den Ausdruck in 525 als störend (S. 196), also nicht von Amos herrührend; auch GUTHE ${ }^{\Sigma}$ sieht darin einen gelehrten Zusatz, ebenso wie in 2 10. $\mathrm{Da}$ GUTHE den Vers aber im.übrigen stehen läßt, so nimmt er an, dab Amos zwar die Tradition von dem Wüstenzuge gekannt habe, aber noch nicht in der Form, daß es gerade 40 Jahre gewesen seien. Mit welchem guten Grunde will man Amos diese Kenntnis absprechen? $\mathrm{Er}$ hat doch so gut wie jeder andere in Israel gewußt, was man sich von der Geschichte der Väter erzahlte, denn er erwähnt ja immer wieder, wie liebevoll Jahwe sein Volk geführt habe von Anfang an. Und wenn nun hier, wie wohl auf Grund der ganzen Zahlenverwertung im AT angenommen werden darf, eine kunstliche Zahl vorliegt, die wie viele andere im ganzen Kalenderwesen ihre Begründung hat ${ }^{2}$, dann werden wir solche Traditionen nicht als jung, sondern als uralt annehmen müssen. Das scheint auch durch die Angaben Dtn 2 I 14 bestätigt zu werden, wo von einem 38jährigen Umziehen des Gebirges Selr die Rede ist, eine Zahl, die sich nur erklären läbt, wenn dem Verfasser die Tradition von 40 Jahren bekannt war und er sich nun bemühte, diese lange Zeit des Wüstenzuges, die ihm selbst unerklärlich war, auf diese Weise verständlich $z$ machen. Wir werden demnach die Kenntnis dieser Tradition dem Amos nicht gut absprechen können.

Um nun über die Echtheit oder Unechtheit von 2 Io entscheiden zu können, müssen wir dieselben Fragen erst für 212 erörtern. MARTI

I Guthe bei KaUtzsch: Das alte Testament 3. Aufl. und bei Sievers und Guthr: Amos, metrisch bearbeitet 1907 .

2 Vgl. A. Jeremias: ATAO2 1906 S. 62. 
behauptet, dieser Vers komme nach der kräftigen abschließenden Frage v. Irb ganz verspatet, passe auch nach v. rra nicht in den Zusammenhang und sei endlich für das Metrum überschüssig (S. I69); Durm schliebt sich (a. a. O. S. 4) diesen Ausführungen MARTIs an.

Gleich der letzte Grund kann wie meist so auch hier nicht als wirklich beweisend für die Unechtheit angesehen werden. Denn die Meinungen uber das Metrum im Hebräischen sind zu verschieden: wo der eine das Metrum für intakt hält, meint ein anderer erst korrigieren zu müssen und ein dritter gar erkennt das Metrum überhaupt nicht an oder teilt zum mindesten den Vers ganz anders ab. Also dieser Grund darf nicht gelten; er wird auch von GUTHE (a. a. O.), NowACK und anderen nicht anerkannt. Und die Behauptung, Amos mübte durchweg nur in metrischer Form gesprochen haben, bedarf noch des Beweises. Außerdem ist es ja auch noch fraglich, ob Amos selbst oder einer seiner Horer die betreffende Stelle niedergeschrieben hat, und dann wird man erst recht nicht so scharf nach dem Metrum fragen dürfen.

Statt der Behauptung aber, v. 12 komme nach der kräftig abschliebenden Frage v. Irb verspatet, läßt sich wohl eher sagen: v. 12 ist die denkbar beste Fortsetzung zu v. 11a, während v. 11b den Zusammenhang zwischen beiden unterbricht. Die Schwierigkeit hat auch GUTHE empfunden, indem er v. ria und v. Irb umstellt; diese Frage ist nur hinter v. 1o oder v. 12 möglich, kann aber besser ganz entbehrt werden.

Amos stellt hier Jahwes Wohltaten an dem Volke und dessen Undank einander gegenuber. Dazu gehört auch, da $\mathrm{Jahwe}$ Israel gewürdigt hat, ihm Männer zu besonderem Dienst zu stellen, daß er sich Nasiräer und Propheten gerade aus diesem Volke erweckt hat. Das war ein besonderer Vorzug, den Israel dankbar hạ̈tte anerkennen müssen, statt dessen aber hat man gerade mit Verachtung diese Taten Jahwes beantwortet: die Nasiräer hat man dazu verführt, ihr Gelubde zu brechen, und den Propheten hat man das Wort verboten.

Was also hier überflüßig, ja geradezu störend ist, das ist die Frage v. IIb; lassen wir diese fort, so haben wir in v. II a und v. I2 die beste Beziehung in chiastischer Stellung; da ist kein Wort zu viel. Gerade v. II a und v. 12 sind wohl bei keinem Propheten so gut zu verstehen wie bei Amos. Er mag damit auf sein eigenes Erlebnis beim Opferfest in Bethel hingewiesen haben. Er war ja auch durchdrungen von dem Gedanken, da $\$ Jahwe es ist, der die Propheten erweckt; denn das hatte er an sich selbst erfahren; um so schwärzer erscheint ihm der Undank des 
Volkes, dessen geistliches Oberhaupt ihn aus dem königlichen Heiligtum in Bethel hinausgewiesen hatte.

Auch v. to erklärt MARTI für unecht, da er sich durch seine prosaische Form und durch seine hinter v. 9 auffallende Stellung als Nachtrag eines Späteren verrate (S. 169). DuHM stimmt ihm auch hierin bei (S. 4).

Wie wenig metrische Gründe gelten können, zeigt sich auch hier wieder darin, daß GUTHE (bei KAUTZSCH) und NowACK ohne Rücksicht auf die prosaische Form den Vers beibehalten. Der zweite Einwand bezüglich der Stellung des Verses geht von dem Gesichtspunkt aus, als ob wir in der Schrift des Amos die Arbeit eines Schriftstellers vor uns hätten, der sich die Gedanken seiner Predigt in ihrer richtigen Aufeinanderfolge gut zurechtgelegt hätte; dann würde allerdings v. 1o zu beanstanden sein. Amos will aber gar kein Schriftsteller sein, er will nicht einmal als Prophet angesehen werden, sondern, was er bis zu seinem Auftreten in Bethel gewesen, das will er auch weiterhin bleiben: ein Hirte und weiter nichts. Die Schrift eines Hirten aber dürfen wir nicht mit dem Mabstabe messen, den MARTI hier anlegt. Amos sprach, wie ihm im Augenblick ums Herz war, und hatte sich seine Predigt nicht vorher ausgearbeitet. Hat er seine Worte selbst niedergeschrieben, dann dürfen wir sie also nicht so scharf beurteilen, und hat sie gar erst einer seiner Hörer aufgezeichnet, dann noch weniger, weil eine so genaue Wiedergabe ja doch nicht möglich war.

Die weitere Behauptung MarTIs, es komme dem Propheten auf den Widerspruch zwischen der Ausrottung der Amoriter durch Jahwe und der Annahme amoritischer Art durch die Israeliten an und da passe die Erwähnung des Auszuges aus Ägypten gar nicht, fallt damit, daß wir v. 12 stehen lassen. Streicht man diesen, dann hat MARTI allerdings Recht. Wie aber oben gezeigt, gehört v. 12 notwendig in den Text und es liegt auch kein triftiger Grund zu seiner Ausschaltung vor. Dann aber ist der in v. 10-12 aufgestellte Gegensatz nicht der von MARTI angegebene, sondern der schon fruher genannte: Jahwes Wohltaten an dem Volk von Anfang an und dessen Undank. Dahin gehört aber auch die Erwähnung des Wüstenzuges. Somit könnte einżig die Stellung des Verses gegen die Echtheit angefuhrt werden. Berücksichtigt man jedoch, was oben über die Herkunft des Amos gesagt ist, dab wir es also mit einem Hirten zu tun haben, dann kann in der Stellung des Verses kein Bedenken gegen seine Echtheit gefunden werden. 


\section{$811-13$.}

In diesen Versen liegt ein Widerspruch vor. V. 11 und 12 sprechen

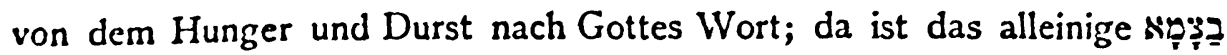
in v. 13 unmöglich, wie schon HITzIG erkannt hat; er hat deshalb v. 13 gestrichen. Welliausen und NowACK dagegen machen den umgckehrten Schluw und streichen v. 11 und 12, weil Amos an eine plötzliche Katastrophe an einem Tage denke, nicht an eine langere Zeit der Gottverlassenheit ₹. MARTI (S. 218f.) und GUTIE (bei KAUTZSCH) gehen noch cinen anderen Weg und schalten nur v. $11 \mathrm{~b}$ und in v. $12 \mathrm{~b}$ die Worte aus, da, wie MARTI meint, ein solches Spielen mit dem Durst einem Amos nicht zugetraut werden könnte; ein Späterer also soll erst durch diese Zusatze die figurliche Deutung hineingebracht haben.

$\mathrm{DaB}$ diese Zerlegung der Verse in echte und unechte Stücke nicht möglich ist, zeigt NowACK, da ja dann die Worte v. IIb und $12 \mathrm{~b}$ von den nach Wasser suchenden Israeliten gemeint wären. Es kann aber bei den beiden Meeren, die da genannt werden, nicht an das galiläische und das Mittelmeer gedacht sein, weil diese wie Jo 220 , Sach 148 anders bezeichnet werden; vielmehr kann nur damit gemeint sein: von einem Ende

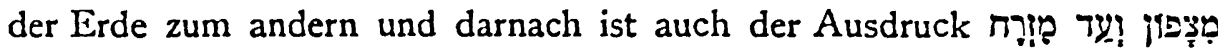
zu verstehen (NowACK 167 f.). Auch GuTHEs Übersetzung in v. 13: „ohne etwas zu finden", zeigt, daß die Ausschaltung von v. 12b nicht möglich

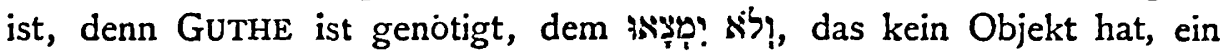

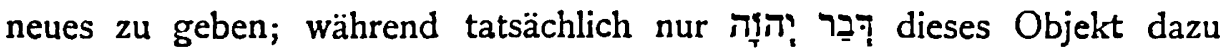
sein kann. Also v. 11b und $12 \mathrm{~b}$ können nicht gestrichen werden. Deswegen nun aber, wie NowACK tut, v. 11 und 12 ganz als Zusatz zu erklaren, ist wohl nicht berechtigt. Auch MARTI gibt zu, daß man darin sehr gut eine Antwort des Amos auf die Abweisung seines prophetischen Wortes durch Amașja in Bethel sehen könnte. Also geben diese Verse für sich genommen keinen Anlaß, sie zu streichen.

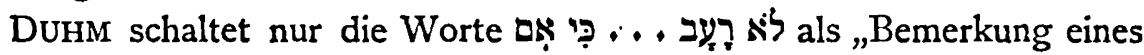

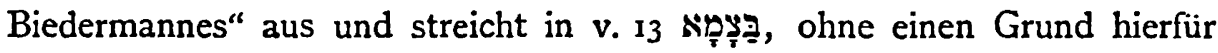
anzugeben (a. a. O. S. 16). DUHM behält also v. II und 12 im wesentlichen als von Amos geschrieben bei, übersieht jedoch bei dieser Korrektur die schon von HITzIG konstatierte Schwierigkeit, daß dann „das Ohnmächtigwerden v. I3 eine starke Hyperbel wäre"; es wäre auch unverständlich, warum gerade "die sorglose Jugend" hier allein genannt sein sollte. So führt auch DuHMs Korrektur noch nicht zum Ziele.

I Wellhausen: Skizzen and Vorarbeiten Heft 3, S. 93 f. Ähnlich Nowack a. a. O. S. 168. 
Der ganze Anstoß, den man an v. $11-13$ nimmt, liegt in dem v. 13 begründet. Dieses Wort steht jedoch an ganz falscher Stelle; es mübte, wenn es ursprünglich wäre, auf das Verbum oder auf הִיז' folgen. Seine unmögliche Stellung am Schluß des Verses aber beweist, daß ein Spàterer, der den Sinn von v. Ir f. falsch verstanden hatte, auf diese Weise das Ohnmächtigwerden noch erklären zu müssen glaubte. Dieses kann unmöglich als die Folge des Durstes nach dem Worte Jahwes gemeint sein, sondern v. 13 gehört hinter v. 1o. Hier wie auch v. 13 ist von בַיצ הַה die Rede, also dem Tage Jahwes, wo so gewaltige Trauer über das ganze Volk kommen wird: da werden selbst diejenigen, die der Stolz

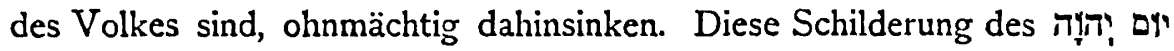
hat nichts zu tun mit der in v. II f., die von "Tagen der Gottverlassenheit" sprechen. Demnach ist v. 13 an v. Io anzuschließen und wohl erst infolge der eben genannten ganz falschen Erweiterung später an die jetzige Stelle gesetzt worden.

V. II 12 und. 14 gehören also zusammen. Hier verkündet Amos wohl mit Beziehung auf sein Erlebnis in Bethel - seinen Zeitgenossen, die jetzt sein Wort, d. i. Jahwes Wort, verachten und sich an die Götzen wenden (v. $14 \mathrm{a}$ ), daß Tage kommen sollen, wo sie nach Jahwe rufen werden, ohne Erhörung zu finden; da wird es mit ihnen $z u$ Ende gehen:

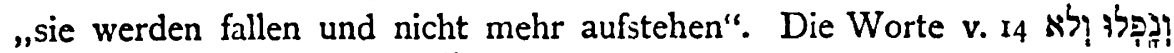
i ip: sind also ebenso wie in dem Bilde 52 ff. $z$ u verstehen. 